\title{
Genomic potential for arsenic efflux and methylation varies among global Prochlorococcus populations
}

\author{
Jaclyn K Saunders and Gabrielle Rocap \\ University of Washington, School of Oceanography, Seattle, WA, USA
}

\begin{abstract}
The globally significant picocyanobacterium Prochlorococcus is the main primary producer in oligotrophic subtropical gyres. When phosphate concentrations are very low in the marine environment, the mol:mol availability of phosphate relative to the chemically similar arsenate molecule is reduced, potentially resulting in increased cellular arsenic exposure. To mediate accidental arsenate uptake, some Prochlorococcus isolates contain genes encoding a full or partial efflux detoxification pathway, consisting of an arsenate reductase (ars $C$ ), an arsenite-specific efflux pump (acr3) and an arsenic-related repressive regulator (arsR). This efflux pathway was the only previously known arsenic detox pathway in Prochlorococcus. We have identified an additional putative arsenic mediation strategy in Prochlorococcus driven by the enzyme arsenite $S$-adenosylmethionine methyltransferase (ArsM) which can convert inorganic arsenic into more innocuous organic forms and appears to be a more widespread mode of detoxification. We used a phylogenetically informed approach to identify Prochlorococcus linked arsenic genes from both pathways in the Global Ocean Sampling survey. The putative arsenic methylation pathway is nearly ubiquitously present in global Prochlorococcus populations. In contrast, the complete efflux pathway is only maintained in populations which experience extremely low $\mathrm{PO}_{4}: \mathrm{AsO}_{4}$, such as regions in the tropical and subtropical Atlantic. Thus, environmental exposure to arsenic appears to select for maintenance of the efflux detoxification pathway in Prochlorococcus. The differential distribution of these two pathways has implications for global arsenic cycling, as their associated end products, arsenite or organoarsenicals, have differing biochemical activities and residence times.

The ISME Journal (2016) 10, 197-209; doi:10.1038/ismej.2015.85; published online 7 July 2015
\end{abstract}

\section{Introduction}

The marine picocyanobacterium Prochlorococcus is the dominant phytoplankter in oligotrophic tropical and subtropical oceans (Campbell et al., 1997; DuRand et al., 2001; Scanlan, 2012). In the subtropical gyres, surface phosphate concentrations are extremely low and in some cases may limit primary production (Wu et al., 2000). Prochlorococcus has evolved several adaptations to this low $\mathrm{P}$ environment, including a high specific affinity for phosphate uptake and low cellular P quotas (Krumhardt et al., 2013). These low cellular P quotas are achieved in part by their small genome sizes (Rocap et al., 2003) and predominance of sulfolipids over phospholipids in the membrane (Van Mooy et al., 2006). As a result, Prochlorococcus under P-replete conditions have a cellular N:P content over 20, well above the Redfield ratio of 16:1, and can exceed 100:1 under P starvation (Bertilsson et al., 2003; Heldal et al., 2003).

Prochlorococcus can be divided up into physiologically and genetically distinct ecotypes (Moore

Correspondence: G Rocap, University of Washington, School of Oceanography, Box 357940, Seattle, 98195 WA, USA.

E-mail: rocap@ocean.washington.edu

Received 15 October 2014; revised 25 February 2015; accepted

3 April 2015; published online 7 July 2015 et al., 1998) which reflect adaptations to environmental parameters like light intensity (Moore et al., 1998; Moore and Chisholm, 1999), temperature optima (Johnson et al., 2006) and nitrogen utilization capabilities (Moore et al., 2002) among others. The multiple strains of Prochlorococcus possess a variable assortment of accessory genes suited to the specific environments they inhabit (Rocap et al., 2003; Kettler et al., 2007). The complement of phosphorus acquisition genes in Prochlorococcus also varies among the strains (Martiny et al., 2006). Many of these genes are found on genomic islands (Coleman et al., 2006) and some have been acquired by horizontal transfer (Rocap et al., 2003). Thus, the occurrence of phosphate acquisition genes is not congruent with 16S rRNA phylogeny (Martiny et al., 2006), but can be correlated with phosphate concentrations in the regions these strains were isolated from. In the field, Prochlorococcus populations in phosphorus-scarce regions contain a greater number of phosphorus acquisition genes, indicating the selective force of nutrient availability on Prochlorococcus genetic capacity (Martiny et al., 2009).

The physiochemical similarities between inorganic phosphate and arsenate can result in the indiscriminate uptake of toxic arsenic into cells by cellular phosphate acquisition systems. The high affinity phosphate uptake system ubiquitously 
present among Prochlorococcus strains (Figure 1) consists of the periplasmic binding protein PstS and the membrane-bound ABC-type transporter PstCAB (Scanlan et al., 2009). The PstCABS system is unable to completely differentiate between arsenate and phosphate in Escherichia coli (Rosenberg et al., 1977; Tawfik and Viola, 2011). Once inside the cell, arsenate can become toxic by competing with phosphate, for example, through the decoupling of oxidative phosphorylation, the process that produces ATP (Mandal and Suzuki, 2002; Oremland and Stolz, 2003). The reduced form, arsenite, is even more toxic because it interferes with enzyme activity by bonding to $-\mathrm{SH}$ and $-\mathrm{OH}$ groups in enzymes (Mandal and Suzuki, 2002; Akter et al., 2005).

Microbes have evolved multiple mechanisms for cellular defense against arsenic, and the genes involved are taxonomically widespread and subject to frequent horizontal transfer (Stolz et al., 2006; Páez-Espino et al., 2009). The general efflux detoxification pathway involves the reduction of arsenate to arsenite, and then subsequent expulsion of arsenic from the cell through arsenite-specific transporters (Carlin et al., 1995; Ghosh et al., 1999). The efflux system, which has evolved independently at least two different times, consists of an arsenate reductase (the analogs ArsC, ACR2 and wzb-like low molecular weight protein tyrosine phosphatase (Bennett et al.,

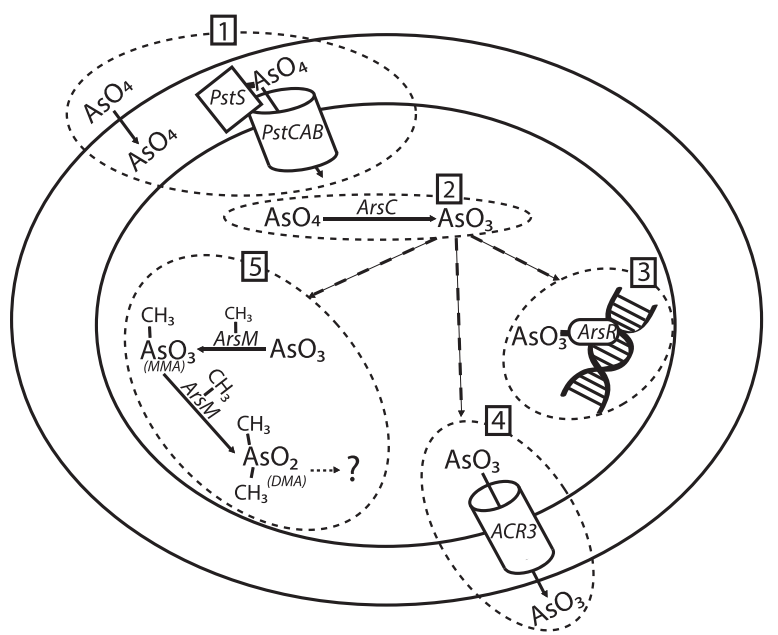

Figure 1 Diagram representing an idealized cross-section of a Prochlorococcus cell highlighting mechanisms likely responsible for arsenic entry into the cell and detoxification. (1) Arsenate likely enters the cell through the inorganic high affinity phosphate transporters PstCABS. (2) Once arsenate is inside the cytoplasm it is reduced by the arsenate reductase ArsC to the (III) oxidation state, arsenite. (3) Arsenite can then bind to the trans-acting ArsRrepressive regulator, (4) arsenite can be recognized by the arsenite efflux transporter ACR3 which shuttles the toxin out of the cell, or (5) the arsenite can be methylated by the putative arsenite $S$-adenosylmethyltransferase, ArsM. Monomethylarsonic acid (MMA) can undergo a series of oxidation and methylation steps to further methylate the species to dimethylarsinic acid (DMA); further methylation into more compound organoarsenicals may also be possible, but cannot be confirmed without laboratory analysis.
2001)) and an arsenite-specific efflux pump (ArsB or ACR3) (Páez-Espino et al., 2009). Additional arsenicrelated genes include an ATPase (ArsA) that couples with ArsB for the expulsion of arsenite from cells, the regulatory elements ArsR and ArsD, and a gene of unknown function arsH (Stolz et al., 2006). The genes sufficient for a complete efflux pathway were previously identified in Prochlorococcus genomes (Scanlan et al., 2009; Figure 1) including the arsenate reductase, ars $C$, the acr3 arsenite efflux transporter and arsR, an arsenite-binding trans-acting repressive regulator (Xu et al., 1996). This efflux detoxification was believed to be the major arsenic detoxification strategy for Prochlorococcus.

A second microbial arsenic detoxification strategy is the methylation of arsenic into more innocuous organic compounds. This pathway involves the repeated methylation and reduction of arsenical compounds to less toxic states mediated by the enzyme ArsM, arsenite $S$-adenosylmethyltransferase (Figure 1) (Qin et al., 2006; Yin et al., 2011). In Rhodopseudomonas palustris, the methylation pathway includes the intermediates monomethylarsonate, and dimethylarsonate, and ultimately results in the production of the gas trimethylarsine oxide, TMAO $(\mathrm{g})$, and subsequent release of arsenic from the cell (Qin et al., 2006). However, the end products of the methylation pathway vary across taxa, and it is not currently possible to determine which organoarsenicals are synthesized through genetic analysis alone (Dembitsky and Levitsky, 2004). TMAO(g) production and presence of arsM genes have been observed in three freshwater cyanobacteria, Microcystis sp. PCC7806, Nostoc sp. PCC7120 and Synechocystis sp. PCC6803 (Yin et al., 2011). A putative arsM was identified in the picocyanobacterium Synechococcus sp. WH 8102 based on sequence similarity and protein binding domain homology (Thomas et al., 2010), but it has not been demonstrated whether or not this species is capable of methylating arsenic in the lab.

In the marine environment, inorganic arsenate accounts for about $85 \%$ of the standing pool of arsenic with the remaining portion of the arsenic pool comprised of the inorganic arsenite and various organoarsenicals, including two of the main products of the methylation pathway, monomethylarsonic acid and dimethylarsinic acid (Andreae, 1979; Cutter et al., 2001; Cutter and Cutter, 2006; Wurl et al., 2013). Arsenate has a nutrient-like depth distribution because it is a bioactive molecule which is transformed by marine biota in the surface waters via the two pathways described above (Andreae, 1979). The degree of uptake and subsequent transformation of arsenate is likely affected by the standing phosphate concentration (Cutter et al., 2001; Cutter and Cutter, 2006). In oligotrophic gyres, the ratio of soluble reactive phosphorus to arsenate can shift dramatically as phosphorus is depleted, resulting in a greater abundance of arsenate relative 
to available phosphate (Karl and Tien, 1997; Wurl et al., 2013). While inorganic arsenic concentrations are relatively stable throughout the surface waters of the open ocean ranging from $12.9-15.7 \mathrm{nmol} \mathrm{l}^{-1}$ (Andreae, 1979; Cutter and Cutter, 1998; Cutter et al., 2001; Ellwood and Maher, 2002; Cutter and Cutter, 2006), surface phosphate concentrations are far more variable, ranging from $<0.1 \mu \mathrm{mol} \mathrm{l^{-1 }}$ in oligotrophic subtropical gyres to $>2.0 \mu \mathrm{mol} \mathrm{l} \mathrm{l}^{-1}$ in the Southern Ocean (Karl, 2007). Thus, the ratio of available $\mathrm{PO}_{4}$ : $\mathrm{AsO}_{4}$ also varies widely across different ocean regions, and may be a major factor driving the risk of competitive arsenic uptake.

To better understand arsenic transformations in marine cyanobacteria, we analyzed arsenic-related genes in Prochlorococcus genomes, and identified a putative arsenite $S$-adenosylmethyltransferase. The evolutionary history of the marine picocyanobacterial arsenic genes was inferred through phylogenetic analysis. To further explore the biogeochemical connection between phosphate scarcity and arsenic transformations in marine ecosystems, we used a phylogenetically informed approach to identify Prochlorococcus-linked arsenic-related genes from the Global Ocean Sampling (GOS) metagenomic dataset spanning various ocean nutrient regimes (Venter et al., 2004; Rusch et al., 2007). The goal of this analysis was to elucidate the putative arsenic-related mechanisms used by Prochlorococcus, and to understand the environmental selective pressures driving the existence of these mechanisms.

\section{Materials and methods}

Identification of Prochlorococcus arsenic genes Arsenic efflux pathway detoxification genes (arsR, $\operatorname{ars} D$, $\operatorname{ars} A, \operatorname{ars} B, \operatorname{acr} 3, \operatorname{ars} C$ and $\operatorname{ars} H$ ) were queried with blastp searches of the Prochlorococcus (taxaid: 1218) portion of NCBI's non-redundant protein sequence database (Altschul et al., 1990) using arsenic detoxification genes from other organisms as queries (Páez-Espino et al., 2009). For genes identified in Prochlorococcus genomes (arsR, ars $C$, acr3) as well as the single copy core genes $g \ln A, \operatorname{rps} D$ and $\operatorname{tyr} S$, the Clusters of Orthologous Groups (COG) associated with blast results were identified (Tatusov et al., 2000; Table 1). The genome browser tool on MicrobesOnline.org (Dehal et al., 2009) was used for identifying genomic context of the various genes within the picocyanobacteria.

To identify a Prochlorococcus arsM, the COGs associated with known functional arsM sequences from other organisms (Qin et al., 2006; Yin et al., 2011) were obtained (COGs 500 and 2226). Sequences of the closely related SAM-dependent mycolic acid cyclopropane synthetase ( $\mathrm{cmaS}$ ) were also included as an outgroup (Yuan and Barry, 1996).

Secondary structure prediction was used to confirm homology of the putatively identified arsM sequence in Prochlorococcus. Sequences identified as arsM in the freshwater cyanobacteria Microcystis sp. PCC7806, Nostoc sp. PCC7120, and Synechocystis sp. PCC 6803 (GenBank accession numbers HQ891147, HQ891148, and HM776638, respectively) were aligned with the putatively identified arsM sequence in Prochlorococcus MED4. All four sequences were submitted individually to the Phyre2 protein prediction server (Kelley and Sternberg, 2009).

Reference alignments and phylogenetic trees

Sequence collection was performed by searching a list of reference organisms (including cyanobacteria, abundant marine bacteria in the GOS metagenomic set (Rusch et al., 2007) and bacterial strains with previously identified arsenic detoxification genes (Páez-Espino et al., 2009)) for genes associated with

Table 1 Presence/absence of arsenic detoxification genes identified within genomes of Prochlorococcus strains

\begin{tabular}{|c|c|c|c|c|c|c|}
\hline & & & $\operatorname{ars} R$ & $a c r 3^{\mathrm{a}}$ & $\operatorname{ars} C$ & $\operatorname{ars} M$ \\
\hline & & & Regulator & Efflux transporter & Reductase & Methyltransferase \\
\hline Strain & Clade & Isolate Region & COG640 & COG798 & COG1393 & COG500 \\
\hline MED4 & High Light 1 & Med. Sea & PMM0714 & PMM0716 & PMM0512 & PMM0416 \\
\hline MIT9515 & High Light 1 & Eq. Pacific & - & - & P9515_05761 & P9515_04771 \\
\hline AS9601 & High Light 2 & Arabian Sea & - & - & A9601 05691 & A9601 04661 \\
\hline MIT9301 & High Light 2 & Sargasso Sea & P9301_12351 & P9301_12581 & P9301_05391 & P9301_04351 \\
\hline MIT9215 & High Light 2 & Eq. Pacific & 二 & 二 & MIT9215 706 & MIT9215 808 \\
\hline MIT9312 & High Light 2 & Gulf Stream & PMT9312_0726 & - & PMT9312_0513 & PMT9312_0411 \\
\hline NATL2A & Low Light 1 & N. Atlantic & PMN2A 0446 & PMN2A 0447 & PMN2A 1845 & - \\
\hline NATL1A & Low Light 1 & N. Atlantic & NATL1 11581 & NATL1 11591 & NATL1_05701 & - \\
\hline SS120 & Low Light 2 & Sargasso Sea & Pro1578 & - & Pro0511 & - \\
\hline MIT9211 & Low Light 3 & Eq. Pacific & - & - & P9211_05131 & P9211 04141 \\
\hline MIT9313 & Low Light 4 & Gulf Stream & PMT1001 & - & PMT1256 & PMT1171 \\
\hline MIT9303 & Low Light 4 & Sargasso Sea & P9303 11041 & P9303 11311 & P9303 07481 & P9303 08531 \\
\hline
\end{tabular}

Abbreviation: COG, Clusters of Orthologous Groups. Presence of gene marked by locus tag, absence represented by '-'. Associated COGs for relevant genes also denoted. Prochlorococcus genomes are listed in order of physiological light optima which follows the 16S rRNA phylogeny. ${ }^{a} a c r 3$ referred to as 'arsB' in Scanlan et al., (2009) and as 'arsA' in Martiny et al. (2006). 
relevant COGs (Table 1) using the online portal www.MicrobesOnline.org (Dehal et al., 2009). The ortholog gene tree tool on www.MicrobesOnline.org was used to generate a list of additional sequences of broader taxonomic range to add greater phylogenetic resolution.

Nucleic acid sequences of the genes were downloaded from www.MicrobesOnline.org and aligned using TranslatorX v 1.1 (Abascal et al., 2010). The amino acid-translated alignments were then used to build phylogenetic reference trees using the program RAxML v 7.2.8 (Stamatakis, 2006). A maximum likelihood tree was inferred taking the best of 20 starting trees using the amino acid substitution matrix model which resulted in the best likelihood score during a trial run (arsR: WAGF; acr3: WAGF; arsC: WAGF; arsM: VTF; glnA: RTREVF; rpsD: RTREVF; tyrS: WAG), empirical character frequencies and a gamma model of rate heterogeneity with RAxML estimated alpha value. Bootstrap analyses were conducted on all trees at $n=100$. These trees, which contained hundreds of taxa (arsR: 295; acr3: 112; arsC: 105; arsM: 289; glnA: 207; rpsD: 99; tyrS: 110), were used for phylogenetic placement of metagenomic reads.

For ease of viewing the broader phylogenetic relationships within the gene trees, smaller trees were constructed. For the genes arsR, ars $C$ and $a c r 3$, a subset of sequences representing the major phylogenetic groups from the original tree were used to construct a smaller tree. For ars $M$, the phylogenetic tree was focused on the clades containing taxa with known arsenite-methyltransferase activity. In addition, arsenite-methyltransferases from eukaryotic algae and mammals were obtained from the OrthoMCL database, which groups orthologous genes from eukaryotic genomes (Chen et al., 2006). For all genes, alignment of the smaller subset of sequences and tree building was similar to the methods described above for the reference trees.

Recruitment and placement of metagenomic reads We analyzed 38 metagenomes from the GOS expedition (Supplementary Table 1). Only sequences from the $0.1-0.8-\mu \mathrm{m}$ filter size fraction were used. For a site to be included in the analysis, we required each single copy core housekeeping gene to be detected at least once to ensure sufficient Prochlorococcus sequence reads. In addition, site GS000a was excluded from analysis because of possible contamination in this sample with non-marine Shewanella and Burkholderia (DeLong, 2005). The unassembled metagenomic sequence reads from the GOS dataset were downloaded from the CAMERA web portal (http://camera.calit2.net; Sun et al., 2011) on 3 Februrary 2011. All work with the dataset was conducted locally.

For each gene $(\operatorname{ars} R, \operatorname{ars} C, \operatorname{acr} 3, \operatorname{ars} M, \operatorname{gln} A, r p s D$ and tyrS), relevant metagenomic reads were recruited with tblastn searches using blastall $\mathrm{v}$ 2.2.25 (Altschul et al., 1997) starting with a Prochlorococcus MED4, MIT9313, NATL2A, SS120 and MIT9312 as well as Synechococcus WH8102 protein sequence as the query (Table 1). Recruited sequences with e-values $<1$ were maintained and analyzed. Sequence reads were trimmed according to their blast alignments to ensure translation in the appropriate reading frame.

For each gene, the recruited GOS reads were aligned to the reference taxa alignment in amino acid space using PaPaRa: Parsimony-based PhylogenyAware Read Alignment program (Berger and Stamatakis, 2011). The metagenomic reads in the $\mathrm{PaPaRa}$ alignment were then assigned to nodes of the reference phylogenetic tree using EPA: Evolutionary Placement Algorithm (Berger et al., 2011). All reads that were placed with appropriate Prochlorococcus clades in the reference trees were identified as 'Prochlorococcus-linked metagenomic reads'. The relative abundance of genes in each metagenomic sample was calculated by normalizing the number of recruited reads to the length of the MED4 query gene and then comparing the length normalized abundance of each arsenic-related gene to the average abundance of length normalized single copy core housekeeping genes. One-way analysis of variance (ANOVA) tests were conducted to ensure that sampling sites within basins could be grouped. Mate pairs from reads identified as Prochlorococcuslinked were tblastn searched against a local nr database (downloaded 4/2013). In addition, the mate pairs identified as Prochlorococcus-linked ars $C$ sequences were recruited to the AS9601 genome using the alignment program Bowtie2 (Langmead and Salzberg, 2012) and visualized with the genomic graphical viewer Tablet (Milne et al., 2013).

\section{Nutrient concentration estimation}

Annual surface statistical average phosphate concentrations were collected from the World Ocean Atlas, 2009 (Garcia et al., 2010), values were averaged over a one degree latitude by one degree longitude square, centered around the coordinates of the metagenomic sample sites except for station GS108a which was averaged over a two by two degree grid owing to the lack of available data (Supplementary Table 1). Minimum surface phosphate concentrations across the same spatial extents were also collected from the World Ocean Atlas, 2009.

\section{Phosphate-binding protein ligand specificity}

Indiscriminate arsenate uptake is likely mediated through the cellular phosphate uptake system which includes the high affinity uptake transporter $p s t S$. To evaluate whether the specificity of the binding sites of the various copies of pstS among Prochlorococcus have changed over time, we submitted pstS sequences to the Phyre2 protein prediction server 
(Kelley and Sternberg, 2009) as well as the 3DLigandSite server (Wass et al., 2010).

\section{Results}

Efflux pathway arsenic detoxification genes in

Prochlorococcus genomes

Prochlorococcus strains vary in their complement of arsenic detoxification genes (Table 1). Arsenate reductase, $\operatorname{ars} C$, which reduces inorganic pentavalent arsenate to the trivalent arsenite state, is present at one copy per genome in all 12 strains for which a genome sequence is available. There is no indication of other known arsenate reductase homologs, such as $a c r 2$ or the arsenate-reductase form of $w z b$ (Bennett et al., 2001) in any strain. In contrast, the efflux transporter acr3 is differentially present among the Prochlorococcus strains, with copies only present in the strains MIT9301, MED4, NATL1A, NATL2A and MIT9303. There are no copies of the alternative ars $B$ form of arsenite transporter among the Prochlorococcus strains. Thus, it appears not all Prochlorococcus have the genetic capacity to expel inorganic arsenite from the cell. The potential for efflux through aquaglycerolporins, which are also leaky to arsenite (Oremland and Stolz, 2003), is also unlikely as no aquaglycerolporins were identified among the Prochlorococcus genomes. The repressive regulator, $\operatorname{ars} R$, is also differentially present among the strains. All strains that possess a copy of acr3 also have the regulator $\operatorname{ars} R$; in addition, ars $R$ is also present in MIT9312, SS120 and MIT9313.

To understand the evolutionary mechanisms responsible for the variable complements of arsenic-related genes in Prochlorococcus, we constructed phylogenetic trees of $\operatorname{ars} C$, ars $R$ and acr3 (Supplementary Figures 1-3) and examined their genomic context. Phylogenetic analysis of all three genes indicates a shared ancestry among the picocyanobacteria. For acr3, the marine picocyanobacteria form a strongly supported monophyletic group. This group is also monophyletic in $\operatorname{ars} C$ and arsR trees, although with less support. For all three genes, the phylogeny within the picocyanobacteria is generally congruent with ribosomal RNA phylogeny. Thus, the arsenic detoxification genes ars $C$, acr3 and ars $R$ are likely ancestral to the picocyanobacteria and the most parsimonious explanation for the differential presence of these genes among Prochlorococcus genomes (Table 1) is multiple independent gene loss events.

Interestingly, within the genomes of strains MED4, NATL1A and NATL2A, the genes acr3 and arsR are in close genomic context to one another and to $\mathrm{P}$ acquisition genes associated with a genomic island (Martiny et al., 2006), and show synteny with each other and with marine Synechococcus. However, in MIT9301 and MIT9303, only arsR is part of the $\mathrm{P}$ island and acr3 is elsewhere. Genomic islands are defined by a preponderance of genes that have been laterally transferred, however, they may also contain ancestral genes through genome rearrangements. In Prochlorococcus genes associated with genomic islands often contribute to specific environmental stress responses (Coleman et al., 2006), it is not surprising to find colocation of arsenic-related genes with phosphorus stress-inducible genes on a genomic island, as the processes of phosphorus stress and arsenic uptake are intertwined. In contrast, in all Prochlorococcus genomes, $\operatorname{ars} C$ is located in a different region of the genome than acr3 and arsR, but this location is syntenous across both Prochlorococcus and Synechococcus. The dispersed location of $\operatorname{ars} C$ in the picocyanobacteria is unusual, as in most bacteria the arsenic detoxification genes are co-located on an ars operon where they are regulated together (Páez-Espino et al., 2009).

\section{Identification of putative Prochlorococcus ArsM}

To determine whether Prochlorococcus also contains the previously unknown arsenic methylation pathway, we attempted to identify an ArsM by constructing an initial phylogenetic tree of a broad array of methyltransferases belonging to COGs 500 and 2226. Within this tree, we identified a clade which includes sequences of known arsenite $S$-adenosylmethyltransferase (arsM) function (Qin et al., 2006; Páez-Espino et al., 2009; Yin et al., 2011) as well as marine picocyanobacterial sequences. A more focused tree was constructed combining sequences from this clade with eukaryotic arsenite $S$-adenosylmethyltransferases (Figure 2). With the exception of Synechococcus WH5701, the putative arsM sequences of the marine picocyanobacteria form a well-supported monophyletic clade among the other ars $M$ sequences. Putative arsM sequences were identified in the majority of Prochlorococcus and Synechococcus genomes, but were not found in Prochlorococcus strains NATL1A, NATL2A or SS120 (Table 1). Interestingly, a few other bacteria, including Synechococcus WH5701, branch within a well-supported clade consisting largely of eukaryotic sequences (from both eukaryotic algae and animals). This suggests that although ars $M$ is ancestral for most of the picocyanobacteria, this gene has been subject to horizontal transfer events.

To further identify whether these Prochlorococcus sequences are likely functioning arsM orthologs, protein structure models were created for the sequence of the putative Prochlorococcus MED4 $\operatorname{ars} M$ as well as sequences of known arsenite $S$-adenosylmethyltransferase function from the freshwater cyanobacteria Nostoc sp. PCC 7120, Synechocystis sp. PCC 6803 and Microcystis aeruginosa NIES843 (Yin et al., 2011). The protein model template c3qnha from the arsM sequence of the eukaryotic red algae Cyanidioschyzon merolae sp. 5508 (Cm-arsM) was identified as the most likely model for all the cyanobacterial sequences at greater than $90 \%$ confidence in homology (Figure 3). 


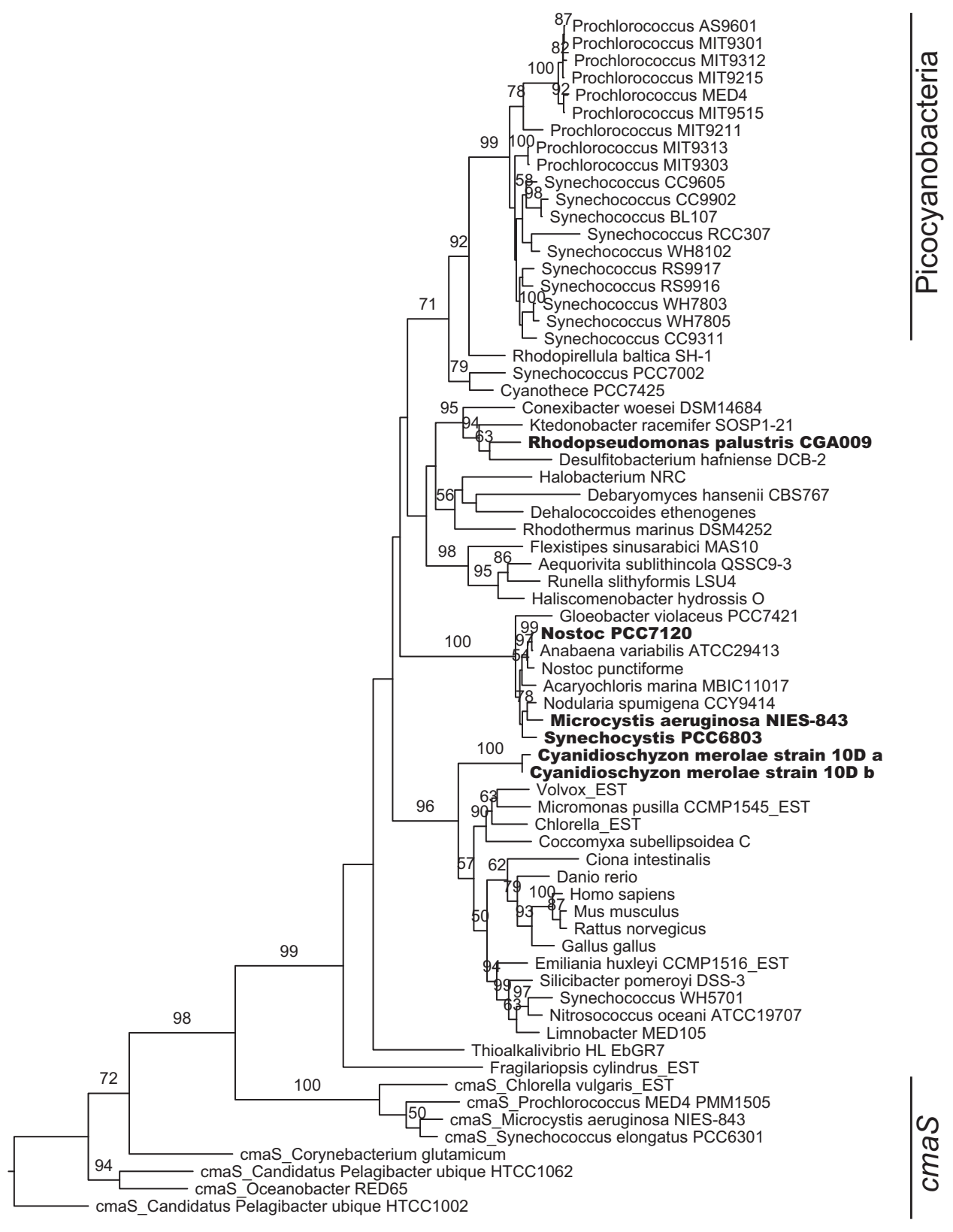

0.8

Figure 2 Phylogeny of arsenite $S$-adenosylmethyltransferases (ars $M$ ). The phylogenetic tree was constructed using the maximumlikelihood program RAxML. The statistical significance of the branch pattern was estimated by conducting a 100 bootstrap replications of the original amino acid alignment; bootstraps $\geqslant 50$ shown. The related $S A M$-dependent mycolic acid cyclopropane synthetase ( $\mathrm{cmaS}$ ) gene was used as an outgroup. Bold sequences represent sequences of genes with confirmed ArsM function (Rosen, 2002; Qin et al., 2006; Yin et al., 2011).

Cysteine residues integral to the binding of arsenite to ArsM protein at positions 72, 174 and 224 in CmArsM (Ajees et al., 2012) are conserved among all the cyanobacterial sequences, including the putative Prochlorococcus MED4 arsM. Overall secondary structure and $S A M$-binding motifs are also conserved. The similarity in these key catalytic residues among the cyanobacteria and the red algae indicate likely conservation of function.
Frequency of arsenic-related genes in wild Prochlorococcus populations

To understand the selective pressures on Prochlorococcus arsenic-related genes in the environment, we examined the frequency of these genes in global Prochlorococcus populations. The Global Ocean Survey sampling sites used for this analysis were predominately from the Sargasso Sea, Caribbean Sea, Eastern Pacific Tropical Upwelling Region and the 

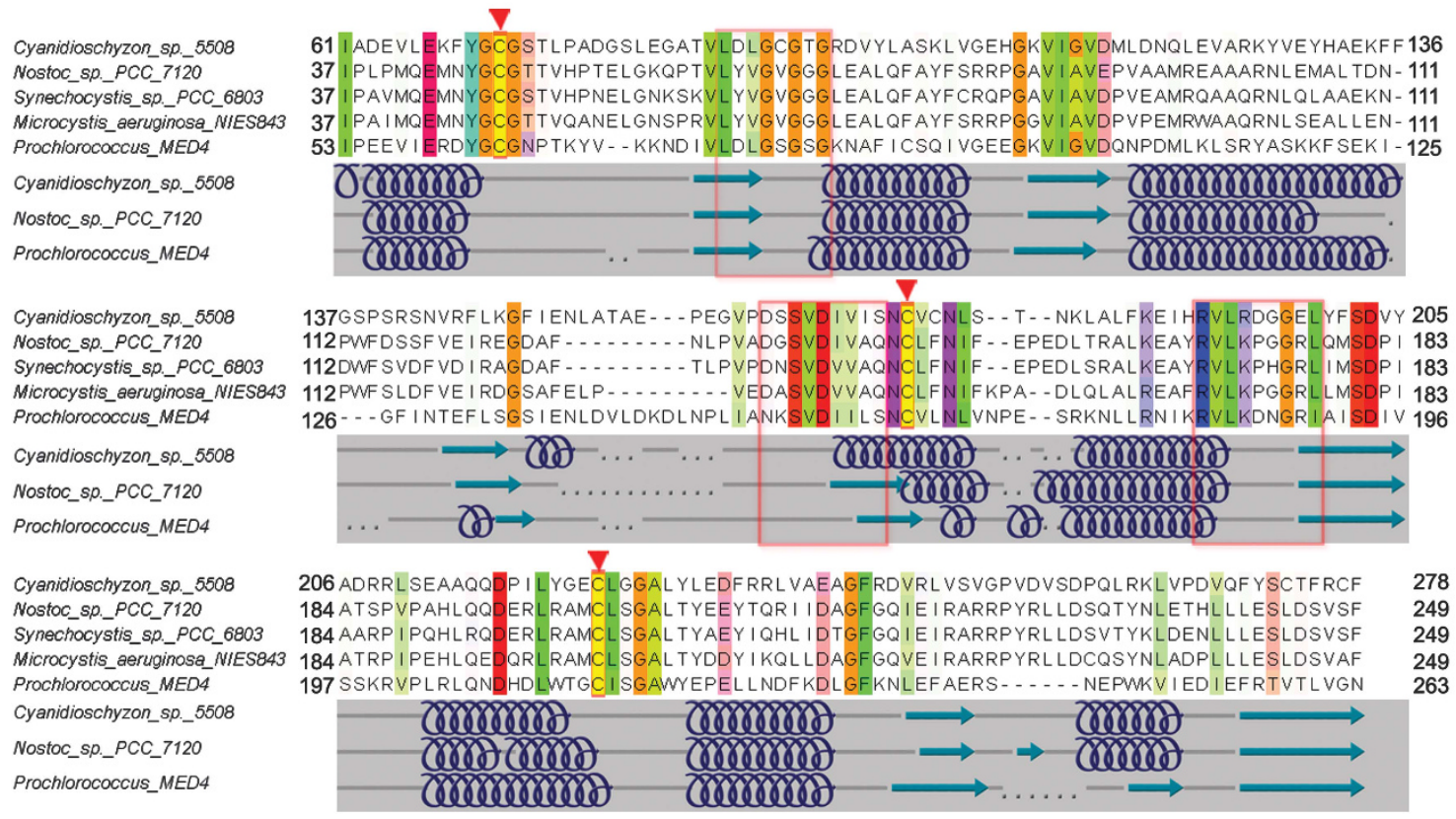

Figure 3 Amino acid pairwise alignment of cyanobacterial sequences to the red algae Cyanidioschyzon merolae sp. 5508 ArsM. Predicted secondary structures generated by the fully automated protein homology/analogy recognition engine Phyre2 (Kelley and Sternberg, 2009) are depicted below the amino acids. Red arrows above amino acid alignment indicate conserved cysteine residues identified to be integral for catalysis of the arsenic methylation reaction in C. merolae (Ajees et al., 2012). Red boxes indicate conserved motifs of regions involved in $S A M$ binding to the protein. Purple loops in predicted secondary structure symbolize alpha helices and teal arrows symbolize predicted beta sheets.

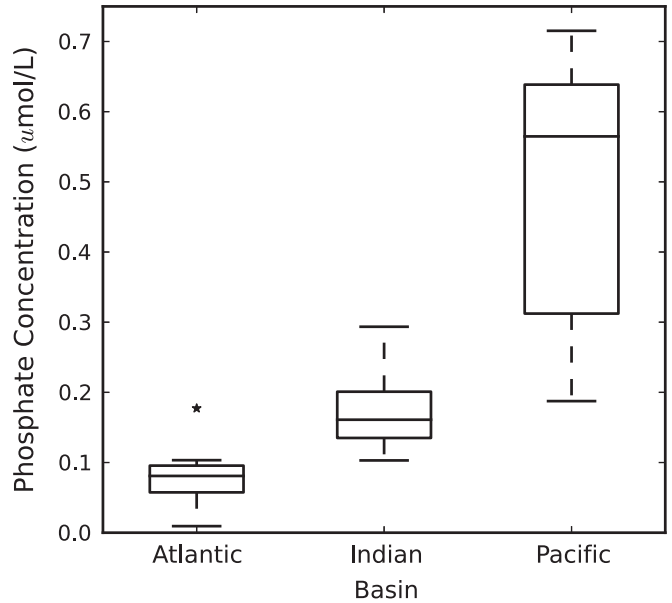

Figure 4 Boxplot of the statistical average annual phosphate concentration at the surface for the GOS metagenome sites analyzed in this study according to ocean basin location. Outliers are represented by stars.

Indian Ocean (Supplementary Table 1). The three oceanic basins sampled captured three significantly different phosphate regimes (Figure 4) (ANOVA $P$-value $<0.000)$. The Atlantic sites have the lowest annual average phosphate conditions $\left(0.08 \mu \mathrm{mol} \mathrm{l^{-1 }}\right)$, the Indian basin sites were higher at $0.18 \mu \mathrm{mol} \mathrm{l}^{-1}$. The Pacific sites had the highest average phosphate concentrations $\left(0.49 \mu \mathrm{mol} \mathrm{l^{-1 }}\right)$ and also the greatest variability among sites. The same pattern of phosphate regimes was identified when comparing phosphate minima, with the Atlantic experiencing the most extreme phosphate minima, the Indian at moderate levels and the Pacific sites experiencing the highest minimum phosphate conditions (ANOVA $P$-value $<0.000$ ). Thus, this dataset effectively captured Prochlorococcus populations exposed to low, moderate and relatively high phosphate conditions.

The presence of arsenic efflux detoxification genes compared with single copy core genes varies among global Prochlorococcus populations (Figure 5). The average occurrence of the single copy core genes $\operatorname{gln} A, r p s D$ and $t y r S$ were used as a proxy for the total number of Prochlorococcus genomes sampled. These genes occur at a rate of one copy per genome among cultured Prochlorococcus (Kettler et al., 2007) and their occurrence in metagenomes did not significantly vary between basins (ANOVA $P$-value for $\operatorname{gln} A, \quad r p s D$ and tyrS: 0.112, 0.056 and 0.105, respectively). The majority of environmental reads for all genes were placed within the high light Prochlorococcus clades. This is not surprising as most of these sites were sampled from 5-m depth or shallower. Among the global Prochlorococcus populations, the arsR regulator occurs at less than one copy per genome on average. The acr3 arsenite efflux transporter also occurs in less than one copy per genome, and was not detected at all in some populations. In contrast, the $\operatorname{ars} C$ arsenate reductase appears to be present at greater than one copy per genome on average in global Prochlorococcus populations. The putative ars $M$ arsenite $S$-adenosylmethyltransferase is present at an average of one 
copy per genome among global Prochlorococcus populations (Figure 5a)

We observed basin scale differences in the relative occurrence of some arsenic detoxification genes (Figure 5, Table 2). In the phosphate-poor Atlantic sites, the ars $R$ regulator appears at about one copy per Prochlorococcus genome (Figure 5b), but it is significantly less frequent in Indian and Pacific populations (ANOVA $P$-value <0.000). acr3, the component of the efflux pathway responsible for expulsion of arsenite from the cell, is present in Atlantic populations at less than one copy per Prochlorococcus genome, still significantly more

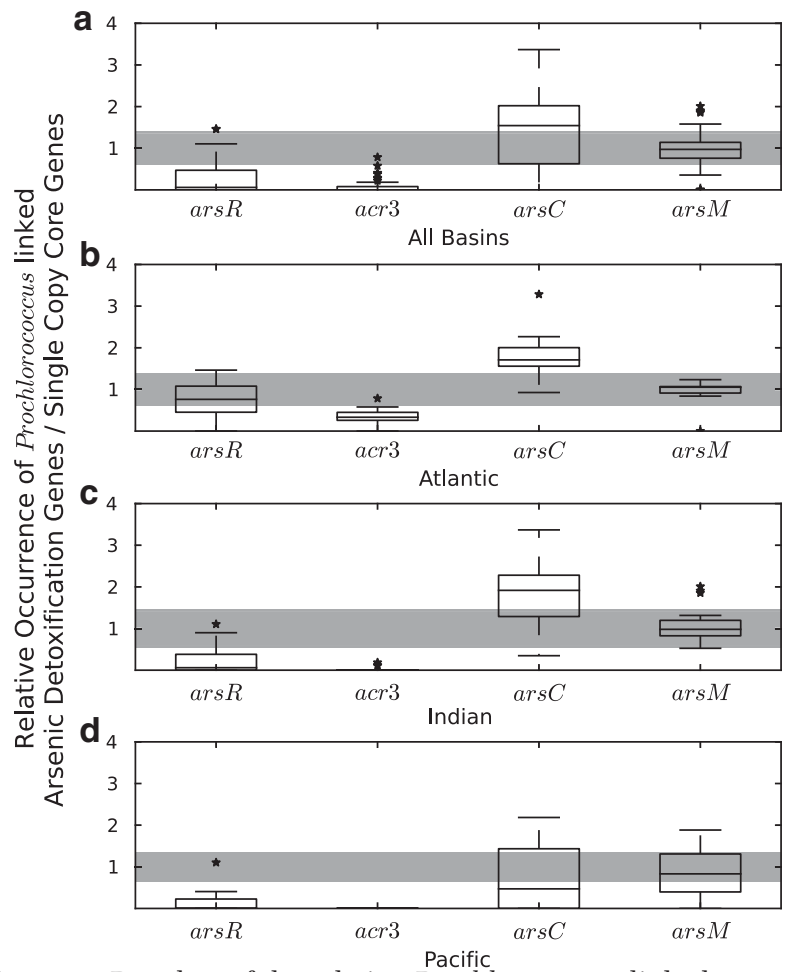

Figure 5 Boxplots of the relative Prochlorococcus-linked arsenic detoxification gene abundance within the GOS metagenomes of the Atlantic, Pacific and Indian basins. The shaded region represents one copy of a gene per genome; the spread of this shading around 1 represents the error associated with the estimation of Prochlorococcus genomes within the basin using single copy core gene abundance. (a) Average across all basins, (b) Atlantic sites, (c) Indian sites and (d) Pacific sites. Outliers are represented by stars.
(ANOVA $P$-value $<0.000$ ) than in Indian and Pacific basin populations, where it was not detected in many samples. In contrast, the ars $C$ arsenate reductase gene occurs in two copies per Prochlorococcus genome in the Atlantic and Indian oceans, but this apparent duplication is not present in Pacific populations. The ars $M$ methyltransferase occurs at about one copy per genome on average in all global Prochlorococcus populations. Thus, Prochlorococcus populations in the Atlantic are capable of arsenic methylation and a portion of the population maintains the genomic potential for arsenite efflux, whereas the Prochlorococcus populations in the Indian and Pacific basins contain the methylation pathway but have lost the capability for arsenite efflux.

\section{Discussion}

Arsenic is the most abundant of environmental toxins with toxicity rooted in its chemical similarity to phosphorus. We suggest that organisms can be susceptible to arsenic toxicity, even at relatively low absolute arsenic concentrations, if phosphorus concentrations are also very low. The marine cyanobacteria Prochlorococcus thrives in one such low $\mathrm{P}$ environment, the tropical and subtropical oligotrophic oceans. Prochlorococcus strains possess differing genomic potentials for arsenic detoxification and the presence/absence pattern of these arsenic-related genes is not congruent with evolutionary relationships based on rRNA genes. Our phylogenetic reconstructions found that the marine picocyanobacteria consistently form a monophyletic clade (Figure 2 and Supplementary Figures 1-3) suggesting that multiple independent gene loss events are responsible for the observed pattern (Table 1). We suggest that the acr3 arsenite efflux transporter and the arsR regulator are being lost where they are no longer needed. Notably, the strains lacking both of these genes (MIT9515, MIT9215, MIT9211 and AS9601) were isolated from either the Equatorial Pacific or the Arabian Sea (Table 1; Rocap et al., 2002) where $\mathrm{P}$ is not typically limiting. They are also the strains observed to have fewest $\mathrm{P}$ acquisition genes (Martiny et al., 2006) indicating

Table 2 Statistical analysis of relative abundance of arsenic detoxification genes in the various ocean basins

Tukey test

ANOVA P-value Partial Eta Squared Atlantic vs Indian Atlantic vs Pacific Indian vs Pacific

\begin{tabular}{|c|c|c|c|c|c|}
\hline ArsR & $0.002 *$ & 0.306 & $0.007^{*}$ & $0.002^{*}$ & 0.737 \\
\hline ArsC & 0.006 * & 0.252 & 0.987 & 0.044 & 0.008 * \\
\hline ACR3 & $0.000^{*}$ & 0.641 & $0.000^{*}$ & $0.000^{*}$ & 0.808 \\
\hline ArsM & 0.355 & 0.057 & N/A & N/A & N/A \\
\hline$\left[\mathrm{PO}_{4}\right]$ & 0.000 * & 0.679 & 0.165 & $0.000 *$ & $0.000^{*}$ \\
\hline
\end{tabular}

Abbreviation: ANOVA, analysis of variance. $P$-values $<0.05$ in bold; $P$-values $<0.01$ with *. A one-way ANOVA was conducted on each gene set, and significantly different gene sets $(P$-value $<0.05)$ were analyzed for pairwise differences between basins using Tukey's post hoc test. 
that the relaxation of pressures induced by low $\mathrm{P}$ has allowed the loss of these genes.

The relative occurrences of arsenic-related genes in Prochlorococcus vary in wild populations as well, where populations from low phosphate regimes maintain a greater genomic potential for arsenic detoxification. The arsenic efflux transporter, and therefore the complete efflux detoxification pathway, is only significantly present in Prochlorococcus populations in the Atlantic Ocean, where annual average phosphate concentrations in the sites sampled are $0.08 \mu \mathrm{mol} \mathrm{l^{-1 }}$. These results are consistent with previous work which found an enrichment of Prochlorococcus-linked acr3 genes at the Bermuda Atlantic Time Series station compared with the Hawaii Ocean Time Series in the North Pacific (Coleman and Chisholm, 2010). ArsR may also be in the process of being lost from some Prochlorococcus populations, as it occurs at less than one copy per genome in the Indian and Pacific basins. We estimated a $\mathrm{PO}_{4}: \mathrm{AsO}_{4}$ ratio as 5:1, 11:1 and 30:1 for the Atlantic, Indian and Pacific sites, respectively. Because data on arsenate concentrations are sparse, this calculation used an arsenate concentration of $15 \mathrm{nmol} \mathrm{l}^{-1}$ (Cutter et al., 2001; Ellwood and Maher, 2002; Cutter and Cutter, 2006) combined with site-specific annual average phosphate concentrations. Thus, the arsenic efflux detoxification pathway is not present in the majority of the Prochlorococcus populations globally, and may be a secondary detoxification pathway that is present only where risk of indiscriminate arsenate uptake is very high, which we suggest occurs when $\mathrm{PO}_{4}: \mathrm{AsO}_{4}$ ratios approach 5:1 or lower. In contrast, the newly identified methylation strategy appears to be a more universal strategy for responding to arsenic in the environment.

Phosphate concentrations low enough to result in P-limitation may also result in enhanced arsenic uptake. The high affinity periplasmic phosphatebinding protein PstS (Figure 1) has an important role in the uptake of arsenate into cells. PstS expression significantly increases under both phosphate limitation and starvation (Martiny et al., 2006; Reistetter et al., 2013) resulting in an increase in phosphate uptake rates compared with P-replete growth conditions (Krumhardt et al., 2013). Thus, in a low $\mathrm{PO}_{4}: \mathrm{AsO}_{4}$ environment, this phosphate stress response, which increases phosphate scavenging, may result in an increase in arsenic uptake. However, it is worth noting that an additional strategy for cellular arsenic defense is the prevention of indiscriminate uptake of arsenate in the first place. Prochlorococcus MIT9313, which lacks the acr3 gene, possesses two copies of pstS, only one of which is expressed in response to phosphate stress (Martiny et al., 2006). Recent work on the two periplasmic phosphate-binding proteins in the extremely arsenic-tolerant Halomonas strain GFAJ-1 from Mono Lake has shown that one of the paralogs can discriminate phosphate from arsenate 4500-fold, compared with 500-fold discrimination of the other copy (Elias et al., 2012). We explored this possibility using protein secondary structure prediction combined with binding site specificity modeling which suggests that the various Prochlorococcus PstS-binding proteins may vary in their ligandbinding specificities. Thus, it is possible that some PstS proteins may have greater capability to discriminate arsenate from phosphate than others, however, additional experiments such as competitive uptake assays would be required to verify this.

Arsenate reductase, $\operatorname{ars} C$, is required by both the efflux pathway and the methylation pathway and is present in a single copy in all isolate Prochlorococcus strains. Strikingly, it appears to be duplicated in environmental Prochlorococcus populations in the Atlantic and the Indian basins, but not in the Pacific, where it occurs at an average of one copy per genome. The duplicated copies of ars $C$ in these populations could confer amplified or differentially regulated expression of arsenate reductase, or may be paralogous genes of another function, although currently there are no known paralogs of this evolutionary form of arsC (Mukhopadhyay et al., 2002). Duplicate copies of an arsenate reductase have been identified in other organisms, including the freshwater cyanobacterium Synechocystis PCC 6803, where they are often found on plasmids (Kaneko et al., 2003, Páez-Espino et al., 2009). It is also possible the arsCs we detected are on cyanophage genomes. Although no arsC-containing cyanophage genomes have been observed, Prochlorococcus phosphate-stress-inducible genes, such as pst $S$ and $p h o H$, are commonly found on cyanophages (Sullivan et al., 2005), and there is evidence that environmental phosphorus conditions are a main selective agent on cyanophage genomes (Kelly et al., 2013). It is also possible that the additional copies represent a very recent horizontal transfer from Prochlorococcus to another organism. However, the associated mate pairs from ars $C$ reads were also identified as Prochlorococcus-like and the majority recruited to the same localized region of a Prochlorococcus genome, thus, at present, the source and function of the additional copies of ars $C$ is unclear.

Prior to this work, the only known arsenic detoxification pathway in Prochlorococcus was the efflux pathway which relies on the ACR3 arsenite efflux transporter (Scanlan et al., 2009). Here, we identified a putative arsenite $S$-adenosylmethyltransferase, $\operatorname{ars} M$, which is present in most sequenced Prochlorococcus strains and appears to be maintained at about one copy per Prochlorococcus genome globally. The presence of ars $M$ indicates that Prochlorococcus likely has the capacity to methylate inorganic arsenicals into less toxic organic forms such as monomethylarsonic acid, dimethylarsinic acid or the gas TMAO(g) and potentially to biosynthesize additional organoarsenical compounds such as arsenolipids and arsenosugars, which are commonly found in eukaryotic algae (Dembitsky and Levitsky, 2004). The widespread 
occurrence of arsM in global Prochlorococcus populations suggests that the methylation pathway may be part of a general metabolic strategy rather than serving solely in arsenic detoxification. For example, Prochlorococcus uses sulfolipids in place of phospholipids in the membrane, thereby reducing the cellular phosphorus demand (Van Mooy et al., 2006) and it is possible that synthesis of arsenolipids allows further reduction of cellular $\mathrm{P}$ quotas. For example, the eukaryotic algae Chlorella methylates inorganic arsenicals and produces polar arsenolipids (Lunde, 1973; Dembitsky and Levitsky, 2004). Arsenosugars are the predominate form of arsenic found in algae, and the freshwater cyanobacteria Synechocystis sp. PCC 6803 and Nostoc sp. PCC 7120 are also capable of producing arsenosugars (Miyashita et al., 2012). Recent work suggests that human ingestion of arsenosugars and arsenolipids might be harmful with potential for release of toxic metabolites with arsenolipids posing the potential for toxicity at levels comparable with inorganic arsenite (Harrington et al., 2008; Raml et al., 2009; Newcombe et al., 2010; Feldmann and Krupp, 2011; Leffers et al., 2013a, b; Meyer et al., 2014). Further laboratory work is needed to verify the functionality of $\operatorname{ars} M$ and to determine to what extent Prochlorococcus is capable of methylating arsenicals and what organic compounds are synthesized.

The ratio of phosphate to arsenate appears to exert influence on the biological conversion of inorganic arsenate to other forms, as the cycling of these two elements are inextricably linked. Along an open ocean transect that covered varying phosphate regimes, when the $\mathrm{PO}_{4}: \mathrm{AsO}_{4}$ ratio was less than 1 , the total arsenic pool consisted of a greater proportion of reduced and methylated arsenicals compared with the relatively phosphate-rich region, with an inorganic $\mathrm{PO}_{4}: \mathrm{AsO}_{4}$ ratio of 100:1 (Cutter and Cutter, 2006). In the North Atlantic, both methylated arsenicals and arsenite detoxification products have been identified and the spatial distribution of arsenite closely corresponded with phosphate availability and can be used in conjunction with measures of alkaline phosphatase to identify regions of phosphorus limitation (Wurl et al., 2013). There is also evidence that the diazotrophic cyanobacterial communities in these phosphate-poor regions are responding to arsenic toxicity. The expression of $\operatorname{ars} C$, ars $A$ and $\operatorname{ars} B$ were detected in a metatranscriptome of a Trichodesmium bloom, suggesting microbial response to arsenic toxicity associated with nitrogen fixation (Hewson et al., 2009). Additionally, expression of the Crocosphaera watsonii WH8105 arsenite efflux transporters increases in laboratory cultures when arsenate is present and the cells are phosphate-stressed. In the oligotrophic Sargasso Sea, with a DIP concentration

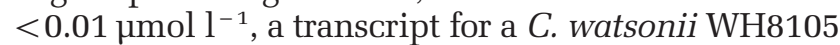
arsenic efflux transporter was successfully identified indicating an in situ response to arsenic toxicity (Dyhrman and Haley, 2011).
The sheer abundance of Prochlorococcus, which can grow at densities greater than $7 \times 10^{5}$ cells $\mathrm{ml}^{-1}$ (Campbell et al., 1998), makes it a key player in biogeochemical cycling of arsenic in the open ocean. As warming associated with climate change causes more intense and persistent stratification of the upper water column, phosphate scarcity and corresponding arsenic exposure will be exacerbated resulting in an increased cycling of arsenicals mediated by Prochlorococcus. The two detoxification pathways have both different end products and intermediary substrates with a broad range of associated toxicities. The previously known but less widespread efflux pathway expels arsenite, which is more toxic than arsenate, from the cell back into the environment where it can either be oxidized back into arsenate in a matter of hours to days (Cutter, 1992) or taken up by eukaryotic organisms, such as algae and fish, via diffusion, hexose permease transporters and aquaporins (Ventura-Lima et al., 2011). The methylation pathway is more effective at sequestering the toxic effects of arsenic, as the detoxified products monomethylarsonic acid and dimethylarsinic acid have a residence time on the order of years rather than days (Cutter and Cutter, 2006), and TMAO(g) may remove arsenic altogether from the marine system. However, the methylation and storage of organoarsenicals by Prochlorococcus may result in bioaccumulation of complexed organoarsenicals in upper trophic levels (Dembitsky and Levitsky, 2004). Prior to the identification of ars , active arsenic detoxification by Prochlorococcus in the environment would presumably only have contributed to the transformation of inorganic arsenate to arsenite, which would then be rapidly oxidized back to arsenate. In addition, this interaction would only occur in the most phosphatestressed regions of the ocean. Our identification of the ars $M$ gene which is widespread among global Prochlorococcus populations suggests Prochlorococcus has a more complicated impact on global arsenic cycling through the production of organoarsenicals.

\section{Conclusions}

Our analysis suggests that Prochlorococcus utilizes arsenic detoxification mechanisms in oceanic regions of phosphorus scarcity where the $\mathrm{PO}_{4}$ : $\mathrm{AsO}_{4}$ ratios are low. There are two detoxification pathways in Prochlorococcus, the efflux pathway (Scanlan et al., 2009) and the putative arsenic methylation pathway identified by this work. The methylation pathway is nearly ubiquitous in global Prochlorococcus populations and likely confers some basal arsenic resistance; however, the efflux pathway is only maintained in populations which experience extreme phosphorus scarcity and have a greater possibility of arsenic uptake into the cell due to reduced $\mathrm{PO}_{4}: \mathrm{AsO}_{4}$ ratios in the environment. Our analysis also suggests that the ability to detoxify 
arsenic is an ancestral state in marine picocyanobacteria. It is vital to understand the products of the different arsenic biochemical pathways, their global occurrence and the rates of detoxification, as the chemical transformations of arsenic by these two pathways are vastly different and have ecological implications for arsenic biogeochemical cycling.

\section{Conflict of Interest}

The authors declare no conflict of interest.

\section{Acknowledgements}

We thank Cedar McKay for help setting up local computational resources. We would also like to thank Simon Berger for providing amino acid support to the PaPaRa alignment program. This work was made possible through support from a National Science Foundation Graduate Research Fellowship to JKS and NSF OCE-1138368 to GR.

\section{References}

Abascal F, Zardoya R, Telford MJ. (2010). TranslatorX: multiple alignment of nucleotide sequences guided by amino acid translations. Nucleic Acids Res 38: W7-W13.

Ajees AA, Marapakala K, Packianathan C, Sankaran B, Rosen BP. (2012). Structure of an As(III) S-adenosylmethionine methyltransferase: insights into the mechanism of arsenic biotransformation. Biochemistry 51: 5476-5485.

Akter KF, Owens G, Davey DE, Naidu R. (2005). Arsenic speciation and toxicity in biological systems. Rev Environ Contam Toxicol 184: 97-149.

Altschul SF, Gish W, Miller W, Myers EW, Lipman DJ. (1990). Basic local alignment search tool. J Mol Biol 215: 403-410.

Altschul SF, Madden TL, Schaffer AA, Zhang J, Zhang Z, Miller W et al. (1997). Gapped BLAST and PSI-BLAST: a new generation of protein database search programs. Nucleic Acids Res 25: 3389-3402.

Andreae MO. (1979). Arsenic speciation in seawater and interstitial waters: the influence of biological-chemical interactions on the chemistry of a trace element. Limnol Oceanogr 24: 440-452.

Bennett MS, Guan Z, Laurberg M, Su XD. (2001). Bacillus subtilis arsenate reductase is structurally and functionally similar to low molecular weight protein tyrosine phosphatases. Proc Natl Acad Sci USA 98: 13577-13582.

Berger SA, Krompass D, Stamatakis A. (2011). Performance, accuracy, and web server for evolutionary placement of short sequence reads under maximum likelihood. Syst Biol 60: 291-302.

Berger SA, Stamatakis A. (2011). Aligning short reads to reference alignments and trees. Bioinformatics 27: 2068-2075.

Bertilsson S, Berglund O, Karl DM, Chisholm SW. (2003). Elemental composition of marine Prochlorococcus and Synechococcus: Implications for the ecological stoichiometry of the sea. Limnol Oceanogr 48: 1721-1731.
Campbell L, Liu H, Nolla HA, Vaulot D. (1997). Annual variability of phytoplankton and bacteria in the subtropical North Pacific Ocean at Station ALOHA during the 1991-1994 ENSO event. Deep Sea Research Part I: Oceanographic Research Papers 44: 167-192.

Campbell L, Landry MR, Constantinou J, Nolla HA, Brown SL, Liu H et al. (1998). Response of microbial community structure to environmental forcing in the Arabian Sea. Deep Sea Research Part II: Topical Studies in Oceanography 45: 2301-2325.

Carlin A, Shi W, Dey S, Rosen BP. (1995). The ars operon of Escherichia coli confers arsenical and antimonial resistance. J Bacteriol 177: 981-986.

Chen F, Mackey AJ, Stoeckert CJ, Roos DS. (2006). OrthoMCL-DB: querying a comprehensive multispecies collection of ortholog groups. Nucleic Acids Res 34: D363-D368.

Coleman ML, Sullivan MB, Martiny AC, Steglich C, Barry K, DeLong EF et al. (2006). Genomic islands and the ecology and evolution of Prochlorococcus. Science 311: 1768-1770.

Coleman ML, Chisholm SW. (2010). Ecosystem-specific selection pressures revealed through comparative population genomics. Proc Natl Acad Sci USA 107: 18634-18639.

Cutter GA. (1992). Kinetic controls on metalloid speciation in seawater. Mar Chem 40: 65-80.

Cutter GA, Cutter LS. (1998). Metalloids in the high latitude North Atlantic Ocean: Sources and internal cycling. Mar Chem 61: 25-36.

Cutter GA, Cutter LS, Featherstone AM, Lohrenz SE. (2001). Antimony and arsenic biogeochemistry in the western Atlantic Ocean. Deep Sea Research Part II: Topical Studies in Oceanography 48: 2895-2915.

Cutter GA, Cutter LS. (2006). Biogeochemistry of arsenic and antimony in the North Pacific Ocean. Geochemistry Geophysics Geosystems 7: 1-12.

Dehal PS, Joachimiak MP, Price MN, Bates JT, Baumohl JK, Chivian D et al. (2009). MicrobesOnline: an integrated portal for comparative and functional genomics. Nucleic Acids Res 38: D396-D400.

DeLong EF. (2005). Microbial community genomics in the ocean. Nat Rev Microbiol 3: 459-469.

Dembitsky VM, Levitsky DO. (2004). Arsenolipids. Prog Lipid Res 43: 403-448.

DuRand MD, Olson RJ, Chisholm SW. (2001). Phytoplankton population dynamics at the Bermuda Atlantic Time-series station in the Sargasso Sea. Deep Sea Research Part II: Topical Studies in Oceanography 48: 1983-2003.

Dyhrman ST, Haley ST. (2011). Arsenate resistance in the unicellular marine diazotroph Crocosphaera watsonii. Front Microbiol 2: 214.

Elias M, Wellner A, Goldin-Azulay K, Chabriere E, Vorholt JA, Erb TJ et al. (2012). The molecular basis of phosphate discrimination in arsenate-rich environments. Nature 491: 134-137.

Ellwood MJ, Maher WA. (2002). An automated hydride generation-cryogenic trapping-ICP-MS system for measuring inorganic and methylated $\mathrm{Ge}, \mathrm{Sb}$ and As species in marine and fresh waters. J Anal At Spectrom 17: 197-203.

Feldmann J, Krupp EM. (2011). Critical review or scientific opinion paper: arsenosugars-a class of benign arsenic species or justification for developing partly speciated arsenic fractionation in foodstuffs? Anal Bioanal Chem 399: 1735-1741. 
Garcia HE, Locarnini RA, Boyer TP, Antonov JI, Zweng MM, Baranova OK et al. (2010). World Ocean Atlas 2009, Volume 4: Nutrients (phosphate, nitrate, silicate). NOAA Atlas NESDIS 71. U.S. Government Printing Office: Washington, D.C, p 398.

Ghosh M, Shen J, Rosen BP. (1999). Pathways of As(III) detoxification in Saccharomyces cerevisiae. Proc Natl Acad Sci USA 96: 5001-5006.

Harrington CF, Brima EI, Jenkins RO. (2008). Biotransformation of arsenobetaine by microorganisms from the human gastrointestinal tract. Chemical Speciation and Bioavailability 20: 173-180.

Heldal M, Scanlan DJ, Norland S, Thingstad F, Mann NH. (2003). Elemental composition of single cells of various strains of marine Prochlorococcus and Synechococcus using X-ray microanalysis. Limnol Oceanogr 48: 1732-1743.

Hewson I, Poretsky RS, Dyhrman ST, Zielinski B, White AE, Tripp HJ et al. (2009). Microbial community gene expression within colonies of the diazotroph, Trichodesmium, from the Southwest Pacific Ocean. ISME J 3: $1286-1300$.

Johnson ZI, Zinser ER, Coe A, McNulty NP, Woodward EM, Chisholm SW. (2006). Niche partitioning among Prochlorococcus ecotypes along ocean-scale environmental gradients. Science 311: 1737-1740.

Kaneko T, Nakamura Y, Sasamoto S, Watanabe A, Kohara M, Matsumoto M et al. (2003). Structural analysis of four large plasmids harboring in a unicellular cyanobacterium, Synechocystis sp. PCC 6803. DNA Res 10: 221-228.

Karl DM, Tien G. (1997). Temporal variability in dissolved phosphorus concentrations in the subtropical North Pacific Ocean. Mar Chem 56: 77-96.

Karl DM. (2007). The Marine Phosphorus Cycle. ASM Press: Washington, pp 523-539.

Kelley LA, Sternberg MJE. (2009). Protein structure prediction on the Web: a case study using the Phyre server. Nat Protoc 4: 363-371.

Kelly L, Ding HM, Huang KH, Osburne MS, Chisholm SW. (2013). Genetic diversity in cultured and wild marine cyanomyoviruses reveals phosphorus stress as a strong selective agent. ISME J 7: 1827-1841.

Kettler GC, Martiny AC, Huang K, Zucker J, Coleman ML, Rodrigue S et al. (2007). Patterns and implications of gene gain and loss in the evolution of Prochlorococcus. PLoS Genet 3: e231.

Krumhardt KM, Callnan K, Roache-Johnson K, Swett T, Robinson D, Reistetter EN et al. (2013). Effects of phosphorus starvation versus limitation on the marine cyanobacterium Prochlorococcus MED4 I: uptake physiology. Environ Microbiol 15: 2114-2128.

Langmead B, Salzberg SL. (2012). Fast gapped-read alignment with Bowtie 2. Nat Meth 9: 357-359.

Leffers L, Ebert F, Taleshi MS, Francesconi KA, Schwerdtle T. (2013a). In vitro toxicological characterization of two arsenosugars and their metabolites. Mol Nutr Food Res 57: 1270-1282.

Leffers L, Wehe CA, Huwel S, Bartel M, Ebert F, Taleshi MS et al. (2013b). In vitro intestinal bioavailability of arsenosugar metabolites and presystemic metabolism of thio-dimethylarsinic acid in Caco-2 cells. Metallomics 5: 1031-1042.

Lunde G. (1973). The synthesis of fat and water soluble arseno organic compounds in marine and limnetic algae. Acta Chem Scand 27: 1586-1594.

Mandal BK, Suzuki KT. (2002). Arsenic round the world: a review. Talanta 58: 201-235.
Martiny AC, Coleman ML, Chisholm SW. (2006). Phosphate acquisition genes in Prochlorococcus ecotypes: Evidence for genome-wide adaptation. Proc Natl Acad Sci USA 103: 12552-12557.

Martiny AC, Huang Y, Li W. (2009). Occurrence of phosphate acquisition genes inProchlorococcuscells from different ocean regions. Environ Microbiol 11: 1340-1347.

Meyer S, Matissek M, Muller SM, Taleshi MS, Ebert F, Francesconi KA et al. (2014). In vitro toxicological characterisation of three arsenic-containing hydrocarbons. Metallomics 6: 1023-1033.

Milne I, Stephen G, Bayer M, Cock PJA, Pritchard L, Cardle L et al. (2013). Using Tablet for visual exploration of second-generation sequencing data. Brief Bioinform 14: 193-202.

Miyashita S-I, Fujiwara S, Tsuzuki M, Kaise T. (2012). Cyanobacteria produce arsenosugars. Environmental Chemistry 9: 474-484.

Moore LR, Rocap G, Chisholm SW. (1998). Physiology and molecular phylogeny of coexisting Prochlorococcus ecotypes. Nature 393: 464-467.

Moore LR, Chisholm SW. (1999). Photophysiology of the Marine Cyanobacterium Prochlorococcus: Ecotypic Differences among Cultured Isolates. Limnol Oceanogr 44: 628-638.

Moore LR, Anton FP, Rocap G, Chisholm SW. (2002). Utilization of different nitrogen sources by the marine cyanobacteria Prochlorococcus and Synechococcus. Limnol Oceanogr 47: 989-996.

Mukhopadhyay R, Rosen BP, Phung LT, Silver S. (2002). Microbial arsenic: from geocycles to genes and enzymes. FEMS Microbiol Rev 26: 311-325.

Newcombe C, Raab A, Williams PN, Deacon C, Haris PI, Meharg AA et al. (2010). Accumulation or production of arsenobetaine in humans? J Environ Monit 12: 832-837.

Oremland RS, Stolz JF. (2003). The ecology of arsenic. Science 300: 939-944.

Páez-Espino D, Tamames J, Lorenzo V, Cánovas D. (2009). Microbial responses to environmental arsenic. BioMetals 22: 117-130.

Qin J, Rosen BP, Zhang Y, Wang G, Franke S, Rensing C. (2006). Arsenic detoxification and evolution of trimethylarsine gas by a microbial arsenite S-adenosylmethionine methyltransferase. Proc Natl Acad Sci USA 103: 2075-2080.

Raml R, Raber G, Rumpler A, Bauernhofer T, Goessler W, Francesconi KA. (2009). Individual variability in the human metabolism of an arsenic-containing carbohydrate, 2',3'-dihydroxypropyl 5-deoxy-5-dimethylarsinoyl-beta-D-riboside, a naturally occurring arsenical in seafood. Chem Res Toxicol 22: 1534-1540.

Reistetter EN, Krumhardt K, Callnan K, Roache-Johnson K, Saunders JK, Moore LR et al. (2013). Effects of phosphorus starvation versus limitation on the marine cyanobacterium Prochlorococcus MED4 II: gene expression. Environ Microbiol 15: 2129-2143.

Rocap G, Distel DL, Waterbury JB, Chisholm SW. (2002). Resolution of Prochlorococcus and Synechococcus ecotypes by using $16 \mathrm{~S}-23 \mathrm{~S}$ ribosomal DNA internal transcribed spacer sequences. Appl Environ Microbiol 68: 1180-1191.

Rocap G, Larimer FW, Lamerdin J, Malfatti S, Chain P, Ahlgren NA et al. (2003). Genome divergence in two Prochlorococcus ecotypes reflects oceanic niche differentiation. Nature 424: 1042-1047. 
Rosen BP. (2002). Biochemistry of arsenic detoxification. FEBS Lett 529: 86-92.

Rosenberg H, Gerdes RG, Chegwidden K. (1977). Two systems for the uptake of phosphate in Escherichia coli. J Bacteriol 131: 505-511.

Rusch DB, Halpern AL, Sutton G, Heidelberg KB, Williamson S, Yooseph S et al. (2007). The Sorcerer II Global Ocean Sampling Expedition: northwest Atlantic through Eastern tropical Pacific. PLoS Biol 5: e77.

Scanlan D. (2012). Marine Picocyanobacteria. In: Whitton BA (ed). Ecology of Cyanobacteria II. Springer: Netherlands, pp 503-533.

Scanlan DJ, Ostrowski M, Mazard S, Dufresne A, Garczarek L, Hess WR et al. (2009). Ecological genomics of marine picocyanobacteria. Microbiol Mol Biol Rev 73: 249-299.

Stamatakis A. (2006). RAxML-VI-HPC: maximum likelihood-based phylogenetic analyses with thousands of taxa and mixed models. Bioinformatics 22: 2688-2690.

Stolz JF, Basu P, Santini JM, Oremland RS. (2006). Arsenic and selenium in microbial metabolism. Annu Rev Microbiol 60: 107-130.

Sullivan MB, Coleman ML, Weigele P, Rohwer F, Chisholm SW. (2005). Three Prochlorococcus cyanophage genomes: Signature features and ecological interpretations. PLoS Biol 3: 790-806.

Sun S, Chen J, Li W, Altintas I, Lin A, Peltier S et al. (2011). Community cyberinfrastructure for Advanced Microbial Ecology Research and Analysis: the CAMERA resource. Nucleic Acids Res 39: D546-D551.

Tatusov RL, Galperin MY, Natale DA, Koonin EV. (2000). The COG database: a tool for genome-scale analysis of protein functions and evolution. Nucleic Acids Res 28: 33-36.

Tawfik DS, Viola RE. (2011). Arsenate replacing phosphate: alternative life chemistries and ion promiscuity. Biochemistry 50: 1128-1134.

Thomas DJ, Nava GM, Cai SY, Boyer JL, Hernandez-Zavala A, Gaskins HR. (2010). Arsenic (+3 oxidation state) methyltransferase and the methylation of arsenicals in the invertebrate chordate Ciona intestinalis. Toxicol Sci 113: $70-76$.

Van Mooy BAS, Rocap G, Fredricks HF, Evans CT, Devol AH. (2006). Sulfolipids dramatically decrease phosphorus demand by picocyanobacteria in oligotrophic marine environments. Proc Natl Acad Sciences USA 103: 8607-8612.

Venter JC, Remington K, Heidelberg JF, Halpern AL, Rusch D, Eisen JA et al. (2004). Environmental genome shotgun sequencing of the Sargasso Sea. Science 304: 66-74.

Ventura-Lima J, Bogo MR, Monserrat JM. (2011). Arsenic toxicity in mammals and aquatic animals: A comparative biochemical approach. Ecotoxicol Environ Saf 74: 211-218.

Wass MN, Kelley LA, Sternberg MJ. (2010). 3DLigandSite: predicting ligand-binding sites using similar structures. Nucleic Acids Res 38: W469-W473.

Wu J, Sunda W, Boyle EA, Karl DM. (2000). Phosphate depletion in the western North Atlantic Ocean. Science 289: 759-762.

Wurl O, Zimmer L, Cutter GA. (2013). Arsenic and phosphorus biogeochemistry in the ocean: Arsenic species as proxies for P-limitation. Limnol Oceanogr 58: 729-740.

Xu C, Shi W, Rosen BP. (1996). The chromosomal arsR gene of Escherichia coli encodes a trans-acting metalloregulatory protein. J Biol Chem 271: 2427-2432.

Yin XX, Chen J, Qin J, Sun GX, Rosen BP, Zhu YG. (2011). Biotransformation and volatilization of arsenic by three photosynthetic cyanobacteria. Plant Physiol 156: 1631-1638.

Yuan Y, Barry CE 3rd. (1996). A common mechanism for the biosynthesis of methoxy and cyclopropyl mycolic acids in Mycobacterium tuberculosis. Proc Natl Acad Sci USA 93: 12828-12833.

Supplementary Information accompanies this paper on The ISME Journal website (http://www.nature.com/ismej) 